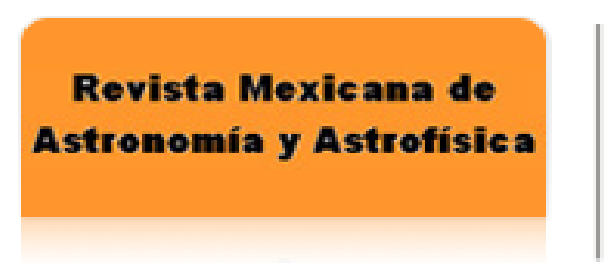

Revista Mexicana de Astronomía y Astrofísica ISSN: 0185-1101

rmaa@astroscu.unam.mx

Instituto de Astronomía

México

Watson, A.M.; De Buizer, J. M.; Radomski, J. T.; Piña, R. K.; Telesco, C. M.

Mid-Infrared Detection of a Hot Molecular Core in G29.96-0.02

Revista Mexicana de Astronomía y Astrofísica, vol. 16, febrero, 2003, pp. 127-130

Instituto de Astronomía

Distrito Federal, México

Available in: http://www.redalyc.org/articulo.oa?id=57116030

How to cite

Complete issue

- More information about this article

Journal's homepage in redalyc.org

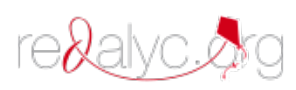

Scientific Information System Network of Scientific Journals from Latin America, the Caribbean, Spain and Portugal Non-profit academic project, developed under the open access initiative 
RevMexAA (Serie de Conferencias), 16, 127-130 (2003)

\title{
MID-INFRARED DETECTION OF A HOT MOLECULAR CORE IN G29.96-0.02
}

\author{
A. M. Watson, ${ }^{1}$ J. M. De Buizer, ${ }^{2}$ J. T. Radomski, ${ }^{3}$ R. K. Piña,${ }^{3}$ and C. M. Telesco ${ }^{3}$
}

\section{RESUMEN}

Se presentan imágenes a 10 y 18 micras del núcleo molecular caliente en la región de formación estelar G29.96-0.02 del interior de la galaxia. Las imágenes son del telescopio de 8 metros de Gemini-North y del instrumento OSCIR de la Universidad de Florida, y tienen una calidad de 0.5 segundos de arco. Tal calidad fue necesario para separar la emisión del núcleo caliente y la de una región H II ultracompacta. Se cree que el núcleo caliente contiene una proto estrella de alta masa, y nuestra detección muestra que los nćleos calientes de la parte interior de la galaxia ya se pueden observar en la región infrarroja media. Eso desvela la posibilidad de estudiar de manera detallada y cuantitativa la formación de estrellas tipo O.

\section{ABSTRACT}

We present 10 and 18 micron images of the hot molecular core in the inner Galaxy star forming region G29.96-0.02. The images were obtained with the University of Florida OSCIR instrument on the GeminiNorth 8 meter telescope and have an image quality of 0.5 arcsecond - this resolution was necessary to separate the hot core from the nearby ultracompact H II region. The hot core is believed to contain a high mass protostar, and our detection shows that inner Galaxy hot cores can indeed be studied at mid-infrared wavelengths. This reveals the possibility of detailed and quantitative investigations of the formation of $\mathrm{O}$ stars.

\section{Key Words: STARS: EARLY-TYPE — STARS: FORMATION}

\section{INTRODUCTION}

Hot molecular cores are compact $(<0.1 \mathrm{pc})$, dense $\left(n>10^{7} \mathrm{~cm}^{-3}\right)$, hot $(50-200 \mathrm{~K})$, and similar in luminosity to $\mathrm{O}$ and $\mathrm{B}$ stars $\left(10^{5}-10^{6} L_{\odot}\right)$. They are often found near ultracompact $\mathrm{H}$ II regions and are often associated with water masers, but free-free radio emission from the hot core itself is observed to be very weak. Taken together, these properties strongly suggest that hot molecular cores harbor very young high mass stars or protostars. As such, they present an opportunity to extend our current hard-won understanding of the formation of stars of one or two solar masses up to the formation of stars of ten and even one hundred solar masses.

Kurtz et al. (1999) reviewed hot cores and gave a table of the derived properties of known examples, including estimates of distances and luminosities. From their table, it is clear all of the local hot cores - those within $2 \mathrm{kpc}$, such as Orion, W3 (OH), DR21 (OH) MM1, and IRAS 20126-have luminosities of roughly $10^{5} L_{\odot}$ and so probably correspond to B stars. While very young B stars are in and of themselves very interesting, $\mathrm{O}$ stars are an order of magnitude more luminous, and for this

\footnotetext{
${ }^{1}$ Universidad Nacional Autónoma de México, Mexico.

${ }^{2}$ Cerro Tololo Inter-American Observatory, Chile.

${ }^{3}$ University of Florida, USA.
}

reason we need to understand both the similarities and differences between the local, lower luminosity hot molecular cores and more distant, higher luminosity hot cores. The higher luminosity hot cores are found in the inner galaxy - an excellent example both of the tendency of more exceptional objects to be more distant and of the central concentration of star formation within our Galaxy.

A preliminary exploration of these sources would involve constraining their spectra sufficiently to measure their luminosity and confirm the suggestion that these have luminosities appropriate for O stars. Further imaging studies could look to locate the heating source, presumably associated with the hottest dust, and confirm that these objects are internally heated (by a protostar) rather than externally heated (by an adjacent ultracompact H II region). Further spectral studies could use the shape of the spectrum to constrain astrophysical models, such as those developed by Osorio, Lizano, \& D'Alessio (1999).

Unfortunately, the luminous inner Galaxy hot molecular cores present a significant problem: following the trend of massive stars to be found in association, they are without exception close to ultracompact H II regions. Observations must therefore separate the emission from the hot core and the H II region, either spectrally or spatially. Since 
ultracompact H II regions do not emit strongly in very highly excited molecular lines, this association does not present a severe problem for molecular studies: Indeed, many of the inner Galaxy hot molecular cores were discovered by Cesaroni, Walmsley, \& Churchwell $(1992)$ in $\mathrm{NH}_{3}(4,4)$ and $(5,5)$ observations with the Effelsberg 100 meter single-dish radio telescope, which gave a beam of roughly 40 arcsec.

However, continuum observations of hot molecular cores require sufficient resolution to adequately separate the H II regions, as both have dust emission that peaks in the far-infared and sub-millimeter and H II regions also have free-free emission. The physical separations between the ultracompact H II region and hot molecular core typically are a few arcseconds, but the resolution required to separate the sources adequately also depends strongly on the contrast between the sources. From the millimeter to the far-infrared regions, resolutions of around one arcsecond are typically required. These can be achieved - just - by current millimeter interferometers, but the beams of current sub-millimeter and far-infrared instruments are at least an order of magnitude too coarse. For this reason, many hot cores have measurements of their dust emission in the Rayleigh tail and nowhere else. The only hope to better constrain the spectrum is observations in the midinfrared, in which array cameras can achieve resolutions of around an arcsecond or better.

\section{CTIO OBSERVATIONS}

Watson \& Mardones (unpublished) observed a large sample of hot molecular cores with the University of Florida OSCIR camera on the CTIO $4 \mathrm{~m}$ telescope at 10 and $18 \mu \mathrm{m}$. Our image quality was about 1 arcsec FWHM. We detected many ultracompact $\mathrm{H}$ II regions, but no hot molecular cores. In many cases, our upper limits for the emission from the hot molecular cores were dominated not by background noise, but by contamination from the ultracompact H II regions. Figures 1 and 2 compare the VLA observations of the G29.96-0.02 hot core in radio continuum and $\mathrm{NH}_{3}(4,4)$ by Cesaroni et al. (1994) with Watson \& Mardones' CTIO $10 \mu \mathrm{m} \mathrm{mi-}$ cron image. The $18 \mu \mathrm{m}$ image is similar. Any midinfrared emission from the hot core is hidden by the wings of the emission from the H II region.

\section{GEMINI OBSERVATIONS}

The obvious next step was to obtain images with better resolution, to try to pull in the emission from

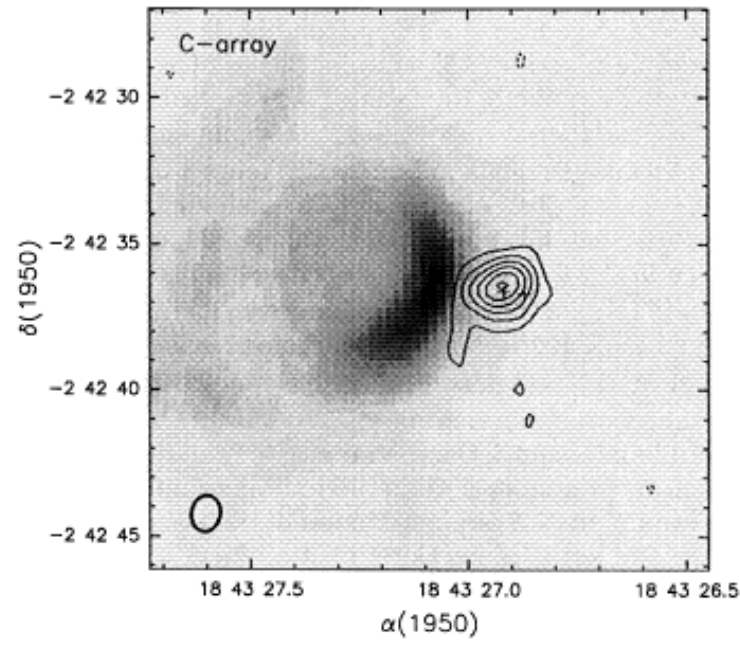

Fig. 1. VLA images of the ultracompact H II region and hot core in G29.96-0.02 by Cesaroni et al. (1994). The gray-scale is $1.3 \mathrm{~cm}$ continuum and is dominated by freefree emission from the cometary H II region. The contours show $\mathrm{NH}_{3}(4,4)$ emission from the hot molecular core in front of the H II region.

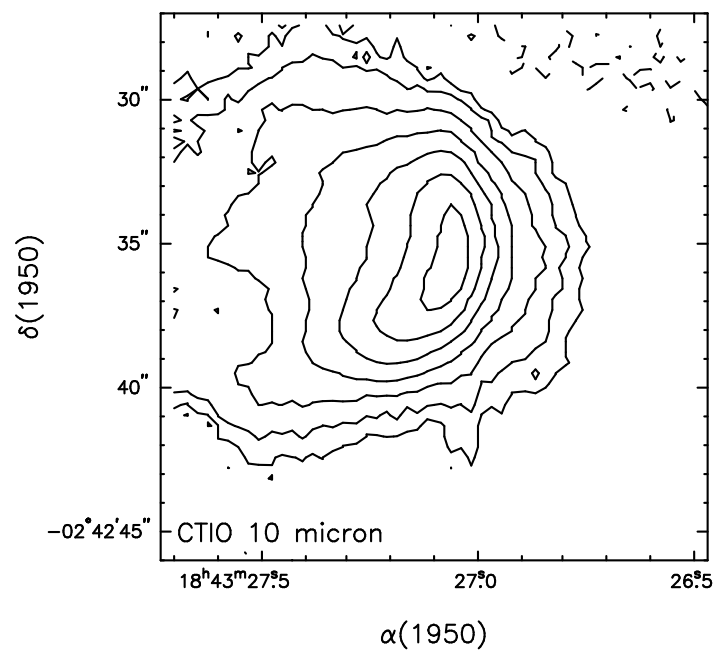

Fig. 2. CTIO/OSCIR 10 micron images of the ultracompact H II region and hot molecular core in G29.96-0.02 (Watson \& Mardones, unpublished). Contours are spaced by factors of 2 from the $\pm 3 \sigma$ level. The position of the hot core lies under the wings of the strong emission from the H II region, and the weak emission from the hot core is swamped. 
the ultracompact $\mathrm{H}$ II region and see the hot molecular core. Figure 3 shows a simple model for the point spread function FWHM for 4 and $8 \mathrm{~m}$ telescopes. The model combines contributions from diffraction and from seeing, which is assumed to fall as $\lambda^{-0.2}$ and is normalized to $0.7 \operatorname{arcsec}$ at $0.55 \mu \mathrm{m}$. Figure 3 shows that diffraction limits the image quality on a $4 \mathrm{~m}$ telescope to $0.8-1.3$ arcsec in the midinfrared $(10-20 \mu \mathrm{m})$ region. Obviously, the effects of diffraction are smaller for a larger telescope, and Figure 3 shows that the image quality of an $8 \mathrm{~m}$ telescope is significantly better, reaching $0.5-0.7$ arcsec, although diffraction is still a significant contributor.

We therefore re-observed G29.96-0.02 with OSCIR on the Gemini North $8 \mathrm{~m}$ telescope (De Buizer et al. 2002), achieving an image quality of around $0.5 \operatorname{arcsec}$ at $10 \mu \mathrm{m}$ and $0.6 \operatorname{arcsec}$ at $18 \mu \mathrm{m}$, in line with the simple model presented above. Our $18 \mu \mathrm{m}$ image is shown in Figure 4. The emission from the hot core stands out from the arc of the ultracompact H II region, although it is still not cleanly separated. The $10 \mu \mathrm{m}$ image is similar, although the hot core is not so prominent.

We analyzed and discussed these image more fully in De Buizer et al. (2002). After subtracting the strong nebular background, we found fluxes for the hot core of $0.1 \mathrm{Jy}$ at $10 \mu \mathrm{m}$ and $2.3 \mathrm{Jy}$ at $18 \mu \mathrm{m}$. These show that the hot core is bright and confirm that only its proximity to the still brighter ultracompact H II region prevented it from being seen it in our CTIO/OSCIR observations. Indeed, with the benefit of hindsight, the hot core can be seen as a slight distortion in the contours of those images.

The lack of a good astrometric calibration of our Gemini data introduces a significant uncertainty in the correspondence between the mid-infrared and radio images. Nevertheless, it seems probable that the mid-IR emission does not coincide precisely with the hot core, which may result from the presence of multiple sources, perhaps at different evolutionary stages, or may simply result from strong and variable extinction toward a single source.

Unfortunately, beyond this we were able to draw very few conclusions, partly because the astrometry is uncertain and partly because the existing millimeter observations (Maxia et al. 2001) do not have adequate resolution to separate the hot core from the ultracompact H II region.

\section{DISCUSSION}

Our observations of G29.96-0.02, while limited by the lack of millimeter data, demonstrate that lu-

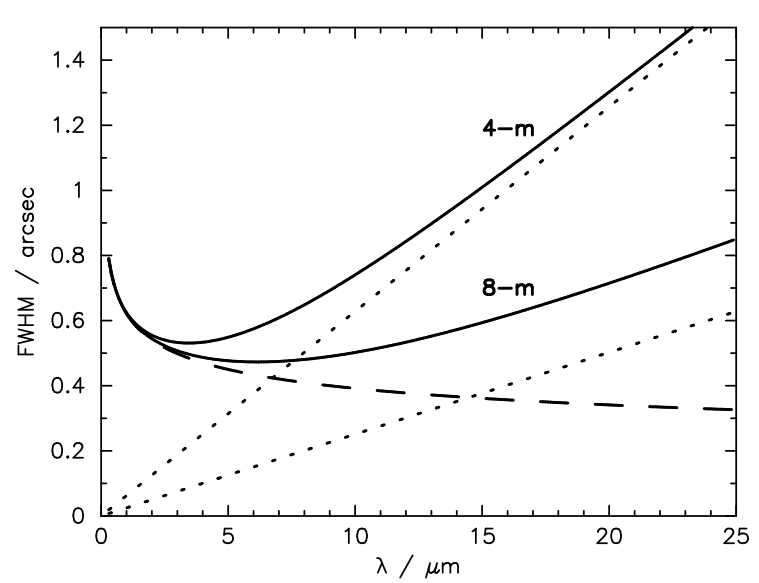

Fig. 3. A simple model for the image quality of 4 and $8 \mathrm{~m}$ telescopes. The model combines diffraction (dotted lines) and seeing (dashed lines; assumed to fall as $\lambda^{-0.2}$ and normalized to 0.7 arcseconds at $0.55 \mu \mathrm{m}$ ). It is apparent that $4 \mathrm{~m}$ telescopes are limited by diffraction to an image quality around 1 arcsec in the mid-infrared, whereas $8 \mathrm{~m}$ telescopes can achieve images closer to 0.5 arcsec.

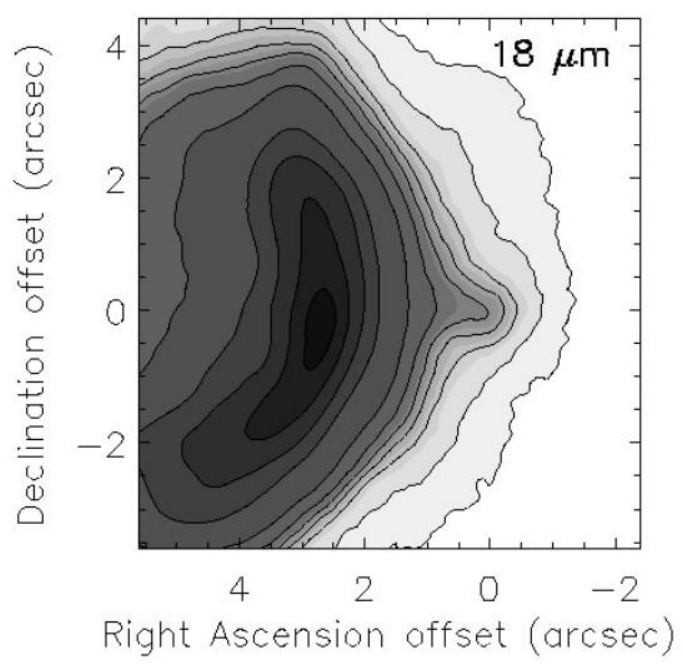

Fig. 4. Gemini/OSCIR $18 \mu \mathrm{m}$ images of the ultracompact H II region and hot molecular core in G29.96-0.02 (De Buizer et al. 2002). The hot core is now clearly seen to the west of the arc of emission from the ultracompact H II region. 
minous, inner Galaxy hot cores can now be observed in the mid-infrared and opens the possibility of obtaining significant advances in the study the formation of $\mathrm{O}$ stars.

To make progress, other sources need to be detected, particularly those for which adequate millimeter observations exist, and to compare these observations to quantitative models, such as those of Osorio et al. (1999). Spectroscopy of the silicate band would be useful, as it can help to separate temperature and optical depth effects.

The problems stemming from uncertainties in the astrometry show that we urgently need to discover how to obtain good astrometry with small field mid-infrared cameras. Perhaps here we need to adapt the techniques to blindly place a spectrograph slit over a very faint object using an offset guider.

\section{REFERENCES}

Cesaroni, R., Churchwell, E., Hofner, P., Walmsley, C. M., \& Kurtz, S. 1994, A\&A, 288, 903

Cesaroni, R., Walmsley, C. M., \& Churchwell, E. 1992, A\&A, 256, 618

De Buizer, J. M., Watson, A. M., Radomski, J. T., Piña, R. K., \& Telesco, C. M. 2002, ApJ, 564, L101

Kurtz, S., Cesaroni, R., Churchwell, E., Hofner, P., \& Walmsley, C. M. 1999, in Protostars and Planets IV, ed. V. Mannings, A. P. Boss, and S . S.Russell(Tucson: University of Arizona Press), 299

Maxia, C., Testi, L., Cesaroni, R., \& Walmsley, C. M. 2001, A\&A, 371, 287

Kroupa, P. 1995, MNRAS, 277, 1491

Osorio, M., Lizano, S., \& D'Alessio, P. 1999, ApJ, 525, 808

A. M. Watson: Instituto de Astronomía, Universidad Nacional Autónoma de México, Apartado Postal 3-72 (Xangari), 58089 Morelia, Michoacán, Mexico (a.watson@astrosmo.unam.mx)

J. M. De Buizer: Cerro Tololo Inter-American Observatory, National Optical Astronomy Observatory, Casilla 603, La Serena, Chile (jdebuizer@ctio.noao.edu)

J. T. Radomski R. K. Piña, and C. M. Telesco: Department of Astronomy, University of Florida, 211 Space Science Research Building, Gainesville, FL 32601, USA (jtr/rpina/telesco@astro.ufl.edu) 